\title{
Limits of zeros of orthogonal polynomials on the circle*
}

\author{
Barry Simon ${ }^{\dagger} \quad$ Vilmos Totik ${ }^{\ddagger}$
}

February 1, 2008

\begin{abstract}
We prove that there is a universal measure on the unit circle such that any probability measure on the unit disk is the limit distribution of some subsequence of the corresponding orthogonal polynomials. This follows from an extension of a result of Alfaro and Vigil (which answered a question of P. Turán): namely, for $n<N$, one can freely prescribe the $n$-th polynomial and $N-n$ zeros of the $N$-th one. We shall also describe all possible limit sets of zeros within the unit disk.
\end{abstract}

\section{Results}

Let $D$ be the open unit disk. We consider Borel measures $d \mu(t), t \in[-\pi, \pi)$ on the unit circle (identified with $\mathbf{R}_{/ \bmod 2 \pi}$ ) of infinite support, and for such a measure let

$$
\Phi_{n}(\mu, z)=z^{n}+\sum_{k=0}^{n-1} c_{n, k}(\mu) z^{k}
$$

be the $n$-th monic orthogonal polynomial.

It is well known that all zeros of $\Phi_{n}$ lie in $D$. The main result of this paper is

Theorem 1 For $1 \leq n<N$, let $\Phi_{n}$ be a monic polynomial of degree $n$ with zeros in $D$ and let there be given $N-n$ points $a_{1}, \ldots, a_{N-n}$ in $D$. Then there is a measure $\mu$ on the unit circle such that $\Phi_{n}(\mu)=\Phi_{n}$ and $a_{i}, i=1, \ldots, N-n$, are zeros (with multiplicity) of $\Phi_{N}(\mu)$.

In short, one can freely prescribe $\Phi_{n}(\mu)$, and $N-n$ zeros of $\Phi_{N}(\mu)$.

We do not know if $\Phi_{N}(\mu)$ is unique, that is, that the other zeros of $\Phi_{N}(\mu)$ are uniquely determined by $\Phi_{n}$ and $a_{1}, \ldots, a_{N-n}$.

\footnotetext{
*For Mathematische Nachrichten issue in memory of F.V. Atkinson

$\dagger$ Supported in part by NSF grant DMS-0140592

${ }^{\ddagger}$ Supported by NSF grant DMS-0097484 and by OTKA T/034323, TS44782
} 
Corollary 2 Let $0=n_{0}<n_{1}<n_{2}<\cdots$ be a sequence of natural numbers and $\left\{a_{j}\right\}_{j=1}^{\infty}$ a sequence in $D$. Then there is a measure $\mu$ such that for each $j=1,2, \ldots$, all $a_{i}, n_{j-1}<i \leq n_{j}$, are zeros (with multiplicity) of $\Phi_{n_{j}}(\mu)$.

Indeed, by induction, we get from Theorem 1 measures $\mu_{j}$ such that

$$
\Phi_{n_{j-1}}\left(\mu_{n_{j-1}}\right)=\Phi_{n_{j-1}}\left(\mu_{n_{j}}\right)
$$

and $a_{i}, n_{j-1}<i \leq n_{j}$, are zeros of $\Phi_{n_{j}}\left(\mu_{n_{j}}\right)$. By a theorem of Geronimus [2], if for an $m$, the $m$-th orthogonal polynomials for two measures coincide, then the same happens for the $l$-th polynomials with all $l \leq m$. Hence we have $\Phi_{n_{k}}\left(\mu_{n_{k}}\right)=\Phi_{n_{k}}\left(\mu_{n_{j}}\right)$ for all $k \leq j$. Since $\Phi_{n_{k}}$ determines the moments up to order $n_{k}$, this shows that for each fixed $m$, the $m$-th moment of the measures $\mu_{n_{k}}, k=1,2, \ldots$, are eventually constant, so the weak-* limit, say $\mu$, of these measures exists and obeys $\Phi_{n_{k}}\left(\mu_{n_{k}}\right)=\Phi_{n_{k}}(\mu)$ for all $k$, and the corollary follows.

To formulate the next corollary, recall that the normalized counting measure $\nu_{n}$ of $\Phi_{n}$ is defined as the measure that puts mass $1 / n$ to each zero of $\Phi_{n}$ (counting multiplicity). We say that a subsequence $\left\{\Phi_{n_{k}}\right\}$ has zero distribution $\nu$ if the normalized counting measures $\nu_{n_{k}}$ converge to $\nu$ in the weak- ${ }^{*}$ topology.

Corollary 3 There is a universal measure $\mu$ on the unit circle such that if $\nu$ is any probability measure on the closed unit disk $\bar{D}$, then some subsequence $\left\{\Phi_{n_{k}}(\mu)\right\}$ has zero distribution $\nu$.

This is immediate from Corollary 2 if we prescribe appropriately $n !-(n-$ $1) !=n !((n-1) / n)$ zeros for $\Phi_{n !}$ in the sequence $\Phi_{0}(\mu), \Phi_{1 !}(\mu), \Phi_{2 !}(\mu), \ldots$ The rest of the proof is standard (take a countable dense subset $\left\{\mu_{j}\right\}$ in the space of probability measures on $\bar{D}$ so that each $\mu_{j}$ occurs infinitely often in this sequence, and select the $n_{k} !\left(\left(n_{k}-1\right) / n_{k}\right)$ zeros for $\Phi_{n_{k}}$ ! so that their distribution converges weak- ${ }^{*}$ to $\mu_{j}$ as we let $n_{k}$ tend to infinity in such a way that $\mu_{j}=\mu_{n_{k}}$ ).

It is known (see, e.g., [11, Lemma 4]) that zeros of orthogonal polynomials cluster to the support $\operatorname{supp}(\mu)$ of the generating measure $\mu$ if the interior of $\operatorname{supp}(\mu)$ is empty and $\mathbf{C} \backslash \operatorname{supp}(\mu)$ is connected. This is due to the fact that the $n$-th orthogonal polynomial minimizes the $L^{2}(\mu)$ norm among all monic polynomials of degree $n$. This is the case, for example, if $\mu$ is a measure on the unit circle, but its support is not the whole circle. The situation changes if the interior of the support is not empty or if $\mathbf{C} \backslash \operatorname{supp}(\mu)$ is not connected. Consider, for example, the closed unit disk or the unit circle and the appropriate (area or arc) Lebesgue measure on it, in which case $\Phi_{n}(z)=z^{n}$, hence all the zeros are at the origin. It was P. Turán who asked if for measures on the unit circle it is possible that the zeros of the orthogonal polynomials cluster to all points of $D$. First, Szabados [8] had shown that for any $\varepsilon>0$, there exists a measure for which the set of limit points of the zeros had area measure $>\pi-\varepsilon$, and then 
Alfaro and Vigil [1] noticed that a positive answer to Turán's problem follows almost immediately from the Szegö recurrence

$$
\Phi_{n+1}(z)=z \Phi_{n}(z)-\bar{\alpha}_{n} \Phi_{n}^{*}(z),
$$

where $\Phi_{n}^{*}(z)=\overline{\Phi_{n}(1 / \bar{z})}$, more precisely from the fact that $\mu \leftrightarrow\left\{\alpha_{n}\right\}_{n=0}^{\infty}$ is a oneto-one correspondence between measures on the circle and $D^{\infty}$ (Verblunsky's theorem [10]). What Alfaro and Vigil showed was that given $\Phi_{n}$, one can prescribe one zero of $\Phi_{n+1}$. Thus, for all $n \geq 1$, one can prescribe exactly one zero $a_{n}$ of $\Phi_{n}$, and this is another one-to-one correspondence $\mu \leftrightarrow\left\{a_{n}\right\}_{n=1}^{\infty}$ between measures on the circle and $D^{\infty}$. Indeed, if $a_{n+1}$ is a zero of $\Phi_{n+1}$, then (2) gives $\overline{\alpha_{n}}=a_{n+1} \Phi_{n}\left(a_{n+1}\right) / \Phi_{n}^{*}\left(a_{n+1}\right)$, and all one needs to know is that $\left|\Phi_{n}(z) / \Phi_{n}^{*}(z)\right|<1$ in $D$ (which follows from the maximum principle for holomorphic functions since on the unit circle, $\left|\Phi_{n}(z)\right|=\left|\Phi_{n}^{*}(z)\right|$, and $\Phi_{n}^{*}$ has all its zeros outside the unit circle).

Let $L_{\mu}$ be the set of limit points of the zeros of all $\Phi_{n}(\mu), n=1,2, \ldots$. This is a subset of $\bar{D}$, and Turán's problem was if it is possible to have $L_{\mu}=\bar{D}$. Our second theorem describes the possible sets for $L_{\mu}$ up to their part on the boundary of $D$.

Theorem 4 Let $F$ be a compact subset of $\bar{D}$. Then there is a measure $\mu$ on the unit circle such that $L_{\mu} \cap D=F \cap D$.

What happens on the boundary is less clear. The proof shows that if $F$ contains the unit circle, then there is a $\mu$ with $L_{\mu}=F$. But not every closed subset $F \subseteq \bar{D}$ is an $L_{\mu}$; for example, $F=\left[\frac{1}{2}, 1\right]$ cannot be the set of limit points. Indeed, suppose to the contrary that $\left[\frac{1}{2}, 1\right]=L_{\mu}$. Then for large $n$, all zeros of $\Phi_{n}(\mu)$ lie in the sector $\arg \left(z-\frac{1}{4}\right)<\frac{1}{4}$, which gives that $\left|\Phi_{n}(z)\right|>\left(\frac{9}{8}\right)^{n}$ for $z$ in a neighborhood $V$ of the point -1 . Therefore, if $\mu(V) \neq 0$, then

$$
\int\left|\Phi_{n}(\mu)\right|^{2} d \mu>c(9 / 8)^{2 n},
$$

which contradicts the fact that the orthogonal polynomials minimize the $L^{2}(\mu)$ norm among all monic polynomials of degree at most $n$ (note that for $z^{n}$, the $L^{2}(\mu)$-norm is at most the total mass of $\mu$ ). Thus, we must have $V \cap \operatorname{supp}(\mu)=\emptyset$, which means that the complement of the support of $\mu$ is connected. But then all points in the support of $\mu$ are limit points of the zeros (see, e.g., [5]), hence the support could only consist of the single point 1 .

We also note that [6] has an alternate way of organizing our proof of Theorem 1.

Derick Atkinson had an important impact on orthogonal polynomials through his book; we are pleased to dedicate this paper to his memory. 


\section{Proof of Theorem 1}

Let $\mathbf{A}=\left(a_{1}, \ldots, a_{N-n}\right) \in D^{N-n}$, and for $\mathcal{Z}=\left(z_{1}, \ldots, z_{n}\right) \in D^{n}$, define

$$
d \mu_{\mathcal{Z}, \mathbf{A}}(t)=d_{\mathcal{Z}, \mathbf{A}}\left(\prod_{j=1}^{n}\left|e^{i t}-z_{j}\right|^{2} \prod_{j=1}^{N-n}\left|e^{i t}-a_{j}\right|^{2}\right)^{-1} d t
$$

where $d_{\mathcal{Z}, \mathbf{A}}$ is a normalizing constant for $\mu_{\mathcal{Z}, \mathbf{A}}$ to have total mass 1 . By Geronimus' theorem [2], $\Phi_{N}\left(\mu_{\mathcal{Z}, \mathbf{A}}\right)$ is the monic polynomial with zeros

$$
z_{1}, \ldots, z_{n}, a_{1}, \ldots, a_{N-n} .
$$

Hence it is enough to show that for some $\mathcal{Z} \in D^{n}$, the coefficients $c_{n, k}\left(\mu_{\mathcal{Z}, \mathbf{A}}\right)$, $k=0, \ldots, n-1$, from (1) are the same as $c_{k}=c_{n, k}\left(\mu_{0}\right)$, where

$$
\Phi_{n}(z)=z^{n}+\sum_{k=0}^{n-1} c_{k} z^{k}
$$

and

$$
d \mu_{0}(t)=\frac{d}{\left|\Phi_{n}\left(e^{i t}\right)\right|^{2}} d t,
$$

with $d$ a normalizing constant. In other words, we want to show that if

$$
\mathcal{M}(\mu)=\left(\Re c_{0}(\mu), \Im c_{0}(\mu), \Re c_{1}(\mu), \Im c_{1}(\mu), \ldots, \Re c_{n-1}(\mu), \Im c_{n-1}(\mu)\right),
$$

then $\mathcal{M}\left(\mu_{\mathcal{Z}, \mathbf{A}}\right)=\mathcal{M}\left(\mu_{0}\right)$.

Set $m_{0}=\mathcal{M}\left(\mu_{0}\right)$ and $F_{\mathbf{A}}(\mathcal{Z})=\mathcal{M}\left(\mu_{\mathcal{Z}, \mathbf{A}}\right)$. We write $z_{j}=x_{j}+i y_{j}$ and we shall also consider $F_{\mathbf{A}}$ as a mapping from $\left(x_{1}, y_{1}, \ldots, x_{n}, y_{n}\right) \in D^{n} \subset \mathbf{R}^{2 n}$ into $\mathbf{R}^{2 n}$, which gives us a continuously differentiable mapping from an open subset $\mathbf{R}^{2 n}$ into $\mathbf{R}^{2 n}$. With these we want to show that the equation $F_{\mathbf{A}}(\mathcal{Z})=m_{0}$ has a solution in $D^{n}$. We shall do that by showing that the topological degree $d\left(F_{\mathbf{A}}, r D^{n}, m_{0}\right)$ of $m_{0}$ with respect to $F_{\mathbf{A}}$ on $r D^{n}$ is not zero for $r<1$ sufficiently close to 1, since then $F_{\mathbf{A}}(\mathcal{Z})=m_{0}$ has a solution in $r D^{n}$ (see [3, Theorem 2.1.1]).

The case $\mathbf{A}=\mathbf{0}$ is instructive, so let us first consider it. Let us also recall (see [3]) that $d\left(F_{\mathbf{0}}, r D^{n}, m_{0}\right)$ is the sum of the sign of the Jacobian $\mathcal{J}_{F_{\mathbf{0}}}$ of $F_{\mathbf{0}}$ (considered as a mapping from $\mathbf{R}^{2 n}$ into $\mathbf{R}^{2 n}$ ) at all points $\mathcal{Z}$ which satisfies the equation $F_{\mathbf{0}}(\mathcal{Z})=m_{0}$ :

$$
d\left(F_{\mathbf{0}}, r D^{n}, m_{0}\right)=\sum_{F_{\mathbf{0}}(\mathcal{Z})=m_{0}} \operatorname{sign}\left(\mathcal{J}_{F_{\mathbf{0}}}\right),
$$

provided none of these $\mathcal{Z}$ is a critical point of $F_{\mathbf{0}}$.

From Szegö's recurrence (2), it is immediate that $\Phi_{n+1}(\mu, z)=z \Phi_{n}(\mu, z)$ if and only if $z=0$ is a zero of $\Phi_{n+1}(\mu, z)$, and a repeated application of this 
gives that $z=0$ is an $N-n$ order zero of $\Phi_{N}(\mu, z)$ if and only if $\Phi_{N}(\mu, z)=$ $z^{N-n} \Phi_{n}(\mu, z)$. In this case, the two associated measures are the same, that is, if

$$
d \mu_{\mathcal{Z}}(t)=d_{\mathcal{Z}}\left(\prod_{j=1}^{n}\left|e^{i t}-z_{j}\right|^{2}\right)^{-1} d t
$$

then $F_{\mathbf{0}}(\mathcal{Z})=\mathcal{M}\left(\mu_{\mathcal{Z}, \mathbf{0}}\right)=\mathcal{M}\left(\mu_{\mathcal{Z}}\right)$. Since by Geronimus' theorem,

$$
\Phi_{n}\left(\mu_{\mathcal{Z}}\right)=\prod_{k}\left(z-z_{k}\right), \quad \mathcal{Z}=\left(z_{1}, \ldots, z_{n}\right),
$$

the coefficient $c_{n, k}\left(\mu_{Z}\right)$ equals $\left((-1)^{n-k}\right.$ times $)$ the $(n-k)$-th elementary symmetric polynomial of the coordinates $z_{j}, j=1, \ldots, n$, of $\mathcal{Z}$. These are $n$ analytic functions $g_{k}\left(z_{1}, \ldots, z_{n}\right), 1 \leq k \leq n$, of the variables $z_{1}, \ldots, z_{n}$, and in this case, the Jacobian $\mathcal{J}_{F_{0}}$ of $F_{\mathbf{0}}$ is the square of the absolute value of the complex Jacobian $\left|\partial g_{k} / \partial z_{j}\right|_{j, k=1}^{n}$ (see, e.g., [4, Lemma 2.1]). Thus, the Jacobian $\mathcal{J}_{F_{\mathbf{0}}}$ is everywhere nonnegative.

Let $Q \subset \mathbf{R}^{2 n}$ be the range of $F_{\mathbf{0}}$. By Geronimus' theorem, $Q$ is the set consisting of (the real and imaginary parts of) the coefficient sequences of all monic polynomials of degree $n$ with zeros in $D$, hence $Q$ is a nonempty open subset of $\mathbf{R}^{2 n} \cdot F_{\mathbf{0}}$ is the map from the zeros of a polynomial to its coefficients, hence it is invariant with respect to permutation of the zeros. Since a polynomial determines its zeros, this map is an $n$ ! cover for those polynomials with distinct zeros. Furthermore, it is a diffeomorphism in a neighborhood of a point of distinct zeros. The set of polynomials with distinct zeros is dense in $Q$, and if we select such a $q \in Q$, then it follows that the topological degree $d\left(F_{\mathbf{0}}, r D^{n}, q\right)$ of $q$ with respect to $F_{\mathbf{0}}$ on $r D^{n}$ is $n$ ! for all $r$ sufficiently close to 1 (so close that $\left.F_{0}^{-1}(\{q\}) \subset r D^{n}\right)$. This is true for a dense set of the $q$ 's in $Q$. Therefore, we actually have $d\left(F_{\mathbf{0}}, r D^{n}, q\right)=n$ ! for every $q \in Q$ for all $r<1$ sufficiently close to 1. Since $m_{0}$ is in the range of this mapping $\left(m_{0}=F_{\mathbf{0}}\left(\mathcal{Z}_{0}\right)\right.$ with $\mathcal{Z}_{0}$ equal to the zero sequence of $\Phi_{n}$ in some order), we have in particular $d\left(F_{\mathbf{0}}, r D^{n}, m_{0}\right)=n$ ! for all $r$ sufficiently close to 1 (so close that $\mathcal{Z}_{0} \in r D^{n}$ ).

This has been the case $\mathbf{A}=\mathbf{0}$, and now we turn to general A. Clearly, $F_{\mathbf{A}}$ is homotopic to $F_{\mathbf{0}}$ under the family $F_{t \mathbf{A}}, t \in[0,1]$, and since the degree is invariant under homotopy if $m_{0}$ is not on the images of the boundary, all that is left is to prove there is an $r$ sufficiently close to 1 such that $m_{0} \notin F_{t \mathbf{A}}\left(\partial\left(r D^{n}\right)\right)$ for all $t \in[0,1]$. In fact, then $d\left(F_{\mathbf{A}}, r D^{n}, m_{0}\right)=n$ !, and hence $F_{\mathbf{A}}(\mathcal{Z})=m_{0}$ has a solution in $r D^{n}$ (see [3, Theorem 2.1.1]).

Suppose to the contrary, that for all $k$, there is an $r_{k}$ with $r_{k} \rightarrow 1, \mathcal{Z}_{k} \in$ $\partial\left(r_{k} D^{n}\right)$, and $t_{k} \in[0,1]$ such that $m_{0}=F_{t_{k}} \mathbf{A}\left(\mathcal{Z}_{k}\right)$. By selecting a subsequence, we may assume that $\mathcal{Z}_{k} \rightarrow \mathcal{Z}^{*} \in \bar{D}^{n}, t_{k} \rightarrow t^{*}$, and $\mu_{\mathcal{Z}_{k}, t_{k} \mathbf{A}} \rightarrow \mu^{*}$, the latter one in the weak-* topology. Then at least one component, say $z_{1}^{*}$, of $\mathcal{Z}^{*}$ is of absolute value 1 and each component $z_{k, j}$ of $\mathcal{Z}_{k}$ converges to the appropriate 
component $z_{j}^{*}$ of $\mathcal{Z}^{*}$ as $k \rightarrow \infty$. Hence for the normalizing constants from (3), we get

$$
\frac{1}{d_{\mathcal{Z}_{k}, t_{k} A_{k}}} \geq \int_{-\pi}^{\pi} \frac{1}{2^{N-1}\left|e^{i t}-z_{k, 1}\right|^{2}} d t=\frac{2 \pi}{2^{N-1}\left(1-\left|z_{k, 1}\right|^{2}\right)} \rightarrow \infty
$$

as $k \rightarrow \infty$. As a consequence, $\mu^{*}$ is supported on $\left\{\theta \in[-\pi, \pi) \mid e^{i \theta} \in \mathcal{Z}^{*} \cap \partial D\right\}$, that is, its support consists of at most $n$ points, and we may assume that $z_{1}^{*} \in \operatorname{supp}\left(\mu^{*}\right)$. If $H_{n-1}$ is any polynomial of degree at most $n-1$, we have

$$
\int \Phi_{n} \overline{H_{n-1}} d \mu_{\mathcal{Z}_{k}, t_{k} \mathbf{A}}=0
$$

for all $k$ (recall that $\Phi_{n}$ is the $n$-th orthogonal polynomial with respect to each $\mu_{\mathcal{Z}_{k}, t_{k} \mathbf{A}}$ by the choice of $\mathcal{Z}_{k}$ ). Hence it follows, by weak-* convergence, that

$$
\int \Phi_{n} \overline{H_{n-1}} d \mu^{*}=0
$$

for any $H_{n-1}$. Now choose $H_{n-1}$ so that it vanishes at all points of the support of $\mu^{*}$ except for $z_{1}^{*}$. Then the previous equality gives

$$
0 \neq \Phi_{n}\left(z_{1}^{*}\right) \overline{H_{n-1}\left(z_{1}^{*}\right)} \mu^{*}\left(\left\{z_{1}^{*}\right\}\right)=\int \Phi_{n} \overline{H_{n-1}} d \mu^{*}=0
$$

and this contradiction shows that, in fact, $m_{0} \neq F_{t \mathbf{A}}\left(\partial r D^{n}\right)$ for all $t \in[0,1]$, provided $r<1$ is sufficiently close to 1 .

\section{Proof of Theorem 4}

Let $D_{r}(z)$ denote the disk of radius $r$ about the point $z$.

We shall use a reasoning similar to the one in [7, Example 2.1.2]. The proof is based on the observation that if $\mu$ is a measure consisting of $m$ mass points and a very small part somewhere else, then $\Phi_{m}(\mu)$ will have precisely one zero close to each mass point (and, of course, no other zero), and $\Phi_{m+1}(\mu)$ will have precisely one zero close to each mass point, plus an additional zero, and this additional zero will be what will move around in the construction to describe the assumed limit set.

Let $F \subseteq \bar{D}$ be the given closed set, and select a countable set $\left\{S_{n}\right\}_{n=0}^{\infty}$ in $D$ such that $F$ is precisely the set of limit points of $\left\{S_{n}\right\}_{n=0}^{\infty}$. Choose also pairwise different points $P_{1}, P_{2}, P_{3}, \ldots$ on the unit circle such that $S_{n}$ lies on the segment joining $P_{2 n+1}$ and $P_{2 n+2}$. It is sufficient to show a measure $\mu$ such that for each $n$, the zeros of $\Phi_{2 n}$ lie very close to $\left\{P_{1}, P_{2}, \ldots, P_{2 n}\right\}$, while the zeros of $\Phi_{2 n+1}$ lie very close to $\left\{P_{1}, P_{2}, \ldots, P_{2 n}\right\} \cup\left\{S_{n}\right\}$. In fact, then the set of limit points of 
the zeros is the closure of the set $\left\{P_{j}\right\}_{j=1}^{\infty} \cup\left\{S_{n}\right\}_{n=1}^{\infty}$, the intersection of which with $D$ is precisely $F \cap D$. The measure $\mu$ will be of the form

$$
\mu=\sum_{n=0}^{\infty} \varepsilon_{n}\left(\beta_{n} \delta_{P_{2 n+1}}+\left(1-\beta_{n}\right) \delta_{P_{2 n+2}}\right),
$$

where $\delta_{P}$ denotes the Dirac mass at the point $P$ and $\beta_{n} \in(0,1), \varepsilon_{n}>0$ will be chosen below.

First of all, we require $\varepsilon_{n+1}<\varepsilon_{n} / 2$ for all $n$. Start with $\varepsilon_{0}=1, \beta_{0}=\frac{1}{2}$. Suppose that we have already selected $\varepsilon_{0}, \ldots, \varepsilon_{n-1}$ and $\beta_{0}, \ldots, \beta_{n-1}$ for some $n>0$. Set

$$
\begin{aligned}
\pi_{n} & =\prod_{j=1}^{2 n}\left|P_{2 n+1}-P_{j}\right|, \\
\kappa_{n} & =\prod_{j=1}^{2 n}\left|P_{2 n+2}-P_{j}\right|,
\end{aligned}
$$

and for a $\beta \in[0,1]$, minimize the expression

$$
\beta \pi_{n}^{2}\left|P_{2 n+1}-s\right|^{2}+(1-\beta) \kappa_{n}^{2}\left|P_{2 n+2}-s\right|^{2}
$$

for $s \in \mathbf{C}$. It is clear that the minimum is taken at some point of the segment connecting $P_{2 n+1}$ and $P_{2 n+2}$, and if $\beta=0$, then it is taken at $P_{2 n+2}$, while if $\beta=1$, then it is taken at $P_{2 n+1}$. As $\beta$ moves from 0 to 1 , there will be a value, which we call $\beta_{n}$, for which the minimum is taken at the point $s=S_{n}$. Now by continuity, there is a $\gamma_{n}>0$ such that if $\left|z_{j}-P_{j}\right|<\gamma_{n}$ for $j=1,2, \ldots, 2 n$ and $T_{2 n}(z)=\prod_{j=1}^{2 n}\left(z-z_{j}\right)$, then the minimum of

$$
\beta_{n+1}\left|T_{n}\left(P_{2 n+1}\right)\right|^{2}\left|P_{2 n+1}-s\right|^{2}+\left(1-\beta_{n+1}\right)\left|T_{2 n}\left(P_{2 n+2}\right)\right|^{2}\left|P_{2 n+2}-s\right|^{2}
$$

is taken somewhere in $D_{1 / n}\left(S_{n}\right)$. We may assume that $\gamma_{n}$ is smaller than $1 / n$ and that it is also smaller than half of the minimal distance in the set

$$
\left\{P_{1}, P_{2}, \ldots, P_{2 n+1}, P_{2 n+2}\right\} .
$$

Thus,

(A) If $\mu$ is a measure such that $\Phi_{2 n+1}(\mu)$ has a zero in each $D_{\gamma_{n}}\left(P_{j}\right), j=$ $1, \ldots, 2 n$, then the remaining zero of $\Phi_{2 n+1}(\mu)$ will be in $D_{1 / n}\left(S_{n}\right)$.

Next, we claim that

(B) There is an $\eta_{n}>0$ such that if $\mu$ is of the form

$$
\mu=\sum_{j=0}^{n-1} \varepsilon_{j}\left(\beta_{j} \delta_{P_{2 j+1}}+\left(1-\beta_{j}\right) \delta_{P_{2 j+2}}\right)+\nu,
$$

where $\nu$ is any measure supported in $\bar{D}$ with total mass $\|\nu\|<\eta_{n}$, then $\Phi_{2 n+1}(\mu)$ has a zero in each $D_{\gamma_{n}}\left(P_{j}\right), j=1, \ldots, 2 n$. 
In fact, we have

$$
\int\left|z \prod_{j=1}^{2 n}\left(z-P_{j}\right)\right|^{2} d \mu \leq 2^{2 n}\|\nu\| \leq 2^{2 n} \eta_{n}
$$

Furthermore, there is a $\rho_{n}>0$ such that for all polynomials $R_{2 n+1}(z)=z^{2 n+1}+$ $\cdots$, the zeros of which omit at least one of $D_{\gamma_{n}}\left(P_{j}\right), j=1, \ldots, 2 n$, we have

$$
\max _{j=1, \ldots, 2 n}\left|R_{2 n+1}\left(P_{j}\right)\right|>\rho_{n} .
$$

Thus, if

$$
\eta_{n}<2^{-2 n} \rho_{n}^{2} \min _{0 \leq j \leq n-1} \varepsilon_{j} \min \left\{\beta_{j}, 1-\beta_{j}\right\},
$$

then no such polynomial can minimize the $L^{2}(\mu)$-norm (recall that $\mu$ has a mass $\geq \varepsilon_{j} \min \left\{\beta_{j}, 1-\beta_{j}\right\}$ at each $\left.P_{2 j}, P_{2 j+1}, j=1, \ldots, n\right)$, and since $\Phi_{2 n+1}(\mu)$ minimizes the $L^{2}(\mu)$-norm, the claim follows.

A perfectly similar argument gives that

(C) There is an $\eta_{n}^{\prime}>0$ such that if $\mu$ is of the form (6) where $\nu$ is any measure supported in $\bar{D}$ with total mass $\|\nu\|<\eta_{n}^{\prime}$, then $\Phi_{2 n}(\mu)$ has a zero in each $D_{\gamma_{n}}\left(P_{j}\right), j=1, \ldots, 2 n$ (and, of course, no other zero).

Now set $\varepsilon_{n}<\min \left(\eta_{n}, \eta_{n}^{\prime}, \varepsilon_{n-1}\right) / 2$. With this choice, the measure from (5) satisfies (B) and (C), and hence we obtain from (A) that we can number the zeros $z_{1}^{(2 n+1)}, \ldots, z_{2 n+1}^{(2 n+1)}$ of $\Phi_{2 n+1}(\mu)$ in such a way that for $j=1,2, \ldots, 2 n$, we have $\left|z_{j}^{(2 n+1)}-P_{j}\right|<1 / n$, and $\left|z_{2 n+1}^{(2 n+1)}-S_{n+1}\right|<1 / n$, and similarly, it follows from (C) that we can number the zeros $z_{1}^{(2 n)}, \ldots, z_{2 n}^{(2 n)}$ of $\Phi_{2 n}(\mu)$ in such a way that for $j=1, \ldots, 2 n$, we have $\left|z_{j}^{(2 n)}-P_{j}\right|<1 / n$, and this is what we wanted to achieve.

\section{References}

[1] M. P. Alfaro and L. Vigil, Solution of a problem of P. Turán on zeros of orthogonal polynomials on the unit circle, J. Approx. Theory, 53 (1988), 195-197.

[2] J. Geronimus, On the trigonometric moment problem, Annals of Math., 47 (1946), 742-761.

[3] N. G. Lloyd, Degree Theory, Cambridge Tracts, 73, Cambridge University Press, Cambridge-New York-Melbourne, 1978. 
[4] R. M. Range, Holomorphic Functions and Integral Representations in Several Complex Variables, Graduate Texts in Mathematics, 108, Springer Verlag, New York, 1986.

[5] E. B. Saff and V. Totik, What parts of a measure's support attract zeros of the corresponding orthogonal polynomials?, Proc. Amer. Math. Soc., 114 (1992), 185-190.

[6] B. Simon, Orthogonal Polynomials on the Unit Circle, AMS Colloquium Series, American Mathematical Society, Providence, RI, expected 2004.

[7] H. Stahl and V. Totik, General Orthogonal Polynomials, Encyclopedia of Mathematics, 43, Cambridge University Press, New York, 1992.

[8] J. Szabados, On some problems connected with polynomials orthogonal on the complex unit circle, Acta Math. Acad. Sci. Hungar., 33 (1979), 197-210.

[9] P. Turán, On some open problems of approximation theory, J. Approx. Theory, 29 (1980), 23-85.

[10] S. Verblunsky, On positive harmonic functions (first part), Proc. London Math. Soc., 40 (1936), 290-320.

[11] H. Widom, Polynomials associated with measures in the complex plane, $J$. Math. Mech., 16 (1967), 997-1013.

Barry Simon

Department of Mathematics

California Institute of Technology

Pasadena, CA 91125

USA

bsimon@caltech.edu

Vilmos Totik

Bolyai Institute

University of Szeged

Szeged

Aradi v. tere 1, 6720, Hungary

and

Department of Mathematics

University of South Florida

4202 E. Fowler Ave, PHY 114

Tampa, FL 33620-5700

USA

totik@math.usf.edu 